\title{
Treatment of Multiple Intracranial Aneurysms with 1-Stage Coiling
}

\author{
P. Jeon, B.M. Kim, D.J. Kim, D.I. Kim, and S.H. Suh
}

\begin{abstract}
BACKGROUND AND PURPOSE: Although multiple intracranial aneurysms are frequent, determining treatment strategy and methods for them is often complicated. The aim of this study was to evaluate the safety and effectiveness of 1-stage coiling for multiple intracranial aneurysms.
\end{abstract}

\begin{abstract}
MATERIALS AND METHODS: All patients who underwent 1-stage coiling for $\geq 2$ aneurysms were identified from a prospectively registered neurointerventional data base during 10 years. The patient characteristics and clinical and angiographic outcomes at discharge and follow-up were retrospectively evaluated.

RESULTS: One hundred sixty-seven patients (male/female ratio, 30:137; mean age, 58 years) with multiple aneurysms (418 aneurysms; mean, 2.5 aneurysms/patient) underwent attempted 1-stage coiling for $\geq 2$ aneurysms ( 359 aneurysms; mean, 2.1 aneurysms/patient). In 131 patients (78.4\%), all detected aneurysms were treated with coiling only. Treatment-related morbidity and mortality at discharge were $1.8 \%$ and $0.6 \%$ per patient, respectively. Of the 132 patients without subarachnoid hemorrhage, 129 (97.7\%) had favorable outcomes (mRS 0-2) at discharge; of the 35 patients with SAH, 27 (77.1\%) had favorable outcomes at discharge. Of the 162 patients (97\%) for whom clinical follow-up was available (mean, 35.8 months), 154 patients (95.1\%) had favorable outcomes. Immediate posttreatment angiography showed complete occlusion in 186 (51.8\%) aneurysms, neck remnants in 134 (37.3\%), sac remnants in 33 (9.2\%), and failure in 6 (1.7\%). Of the 262 (73.9\%) aneurysms that underwent follow-up imaging (mean, 24.8 months), 244 (93.1\%) showed a stable or improved state, with 12 (4.6\%) minor and $6(2.3 \%)$ major recurrences.
\end{abstract}

CONCLUSIONS: One-stage coiling of multiple aneurysms seems to be safe and effective, with low morbidity and mortality.

$I^{\mathrm{n}}$ tracranial aneurysm is the most important cause of subarachnoid hemorrhage, resulting in $8 \%-20 \%$ dependent morbidity and $37 \%-57 \%$ mortality. ${ }^{1}$ Strategies and methods for treating intracranial aneurysms have been extensively studied. Multiple intracranial aneurysms are frequent, with a reported incidence of $19 \%-34 \%$ of patients who present with $\mathrm{SAH}^{2-5}$ Determining treatment strategy and methods for multiple aneurysms is often complicated. Coiling is currently accepted as a standard treatment for ruptured or unruptured intracranial aneurysms and has been increasingly used, regardless of the location of the intracranial

Received August 24, 2013; accepted after revision October 21.

From the Department of Radiology (P.J.), Samsung Medical Center, Sungkyunkwan University School of Medicine, Seoul, Korea; Department of Radiology (B.M.K D.J.K., D.I.K.), Severance Hospital, and Department of Radiology (S.H.S.), Gangnam Severance Hospital, Yonsei University College of Medicine, Seoul, Korea.

This study was supported by a faculty grant of Yonsei University College of Medicine for 2009 (6-2009-0080).

Please address correspondence to Byung Moon Kim, MD, PhD, Department of Radiology, Severance Hospital, Yonsei University College of Medicine, 50 Yonsei-ro, Seodaemun-gu, Seoul 120-752, Korea; e-mail: bmoon21@hanmail.net

http://dx.doi.org/10.3174/ajnr.A3821 aneurysm. ${ }^{6}$ Nevertheless, while many cases of clipping for multiple aneurysms have been reported, ${ }^{7-12}$ there were only 2 small case series using coiling for multiple aneurysms. ${ }^{13,14}$ The purpose of this study was to evaluate the safety and effectiveness of 1-stage coiling for multiple intracranial aneurysms.

\section{MATERIALS AND METHODS}

The institutional review board approved this retrospective analysis and waived patient informed consent due to the study design. All patients who underwent 1-stage coiling for $\geq 2$ aneurysms were identified from a prospectively registered neurointerventional data base in 2 tertiary referral academic hospitals from January 2003 through December 2012. In patients with acute SAH with multiple aneurysms, the ruptured aneurysm was first treated; then, if feasible, the other aneurysms were treated in the same session. However, if it was not clear which aneurysm was ruptured, coiling was attempted only if it was feasible for all potentially ruptured aneurysms. In the patients with multiple unruptured aneurysms, neurointerventionists and vascular neurosurgeons determined, after diagnostic angiography, which aneurysms could be treated with 1-stage coiling. All procedures 
Characteristics of patients and aneurysms, and immediate clinical and angiographic outcomes after treatment with 1-stage coiling ${ }^{a}$

\section{Characteristics and Outcomes}

\begin{tabular}{|c|c|}
\hline No. of patients (No. of aneurysms coiled) & $167(359)$ \\
\hline Age (yr) (mean) (range) & $57.7 \pm 10.6(30-83)$ \\
\hline Male/female & 30:137 \\
\hline Presentation with subarachnoid hemorrhage & $35(21.0 \%)$ \\
\hline No. of aneurysms per patient (range) & $2.5 \pm 0.9(2-7)$ \\
\hline No. of coiled aneurysms per patient (range) & $2.1 \pm 0.4(2-3)$ \\
\hline No. of patients with all detected aneurysms coiled & $131(78.4 \%)$ \\
\hline \multicolumn{2}{|l|}{ Location of aneurysms coiled ( $n=359)$} \\
\hline ICA paraclinoid & $135(37.6 \%)$ \\
\hline ICA-posterior communicating artery & $56(15.6 \%)$ \\
\hline ICA-anterior choroidal artery & $26(7.2 \%)$ \\
\hline ICA bifurcation & $7(1.9 \%)$ \\
\hline Middle cerebral artery & $58(16.2 \%)$ \\
\hline Anterior cerebral artery & $44(12.3 \%)$ \\
\hline Vertebrobasilar artery & $32(8.9 \%)$ \\
\hline Aneurysm size (mm) (mean) (range) & $5.6 \pm 2.7(2-21)$ \\
\hline$<10 \mathrm{~mm}$ & $326(90.8 \%)$ \\
\hline $10-24.9 \mathrm{~mm}$ & $33(9.2 \%)$ \\
\hline Neck size (mm) (mean) (range) & $3.3 \pm 1.3(0.4-10.0)$ \\
\hline Wide neck (neck size $\geq 4 \mathrm{~mm}$ or dome-to-neck ratio $<1.5$ ) & $233(64.9 \%)$ \\
\hline \multicolumn{2}{|l|}{ Distribution of aneurysms coiled } \\
\hline Unilateral anterior circulation & $73(43.7 \%)$ \\
\hline Bilateral anterior circulation & $66(39.5 \%)$ \\
\hline Posterior circulation & $3(1.8 \%)$ \\
\hline Unilateral anterior + posterior circulation & $20(12.0 \%)$ \\
\hline Bilateral anterior + posterior circulation & $5(3.0 \%)$ \\
\hline Treatment-related morbidity $(n=167)$ & 3 (1.8\%; 95\% Cl, 0.24-3.83\%) \\
\hline Treatment-related mortality $(n=167)$ & $1(0.6 \% ; 95 \% \mathrm{Cl}, 0.58-1.78 \%)$ \\
\hline \multicolumn{2}{|l|}{ Immediate angiographic outcome } \\
\hline Complete occlusion & $186(51.8 \%)$ \\
\hline Neck remnant & $134(37.3 \%)$ \\
\hline Sac remnant & $33(9.2 \%)$ \\
\hline Coiling failure & $6(1.7 \%)$ \\
\hline
\end{tabular}

${ }^{a}$ Data are shown as number (\%) except where otherwise noted.

were performed with the patient under general anesthesia. Coiling was performed by using a conventional single catheter, multicatheter, balloon-assisted, stent-assisted, or combined technique. For all unruptured aneurysms, preoperative dual antiplatelet medication (aspirin, 100-325 mg, and clopidogrel, 75 $\mathrm{mg}$ ) was given at least 5 days before treatment for all patients starting in late 2010. Generally, dual antiplatelet medication was stopped after the treatment but was maintained for at least 3 months and then changed to aspirin monotherapy for patients who received stent-assisted coiling.

All clinical and radiographic data were prospectively registered into neurointerventional and neurosurgical data bases in each hospital at the time of treatment and then were retrospectively analyzed by the authors. All periprocedural complications during and after treatment were prospectively recorded and retrospectively evaluated for this study. For patients presenting with $\mathrm{SAH}$, treatment-related morbidity was defined as treatment-related complications causing deterioration of the patient's neurologic condition. For patients without SAH, treatment-related morbidity was defined as any objective clinical symptom developing after coiling and remaining at discharge. Patients were regularly followed up on an outpatient basis, according to the protocol of each institution, typically 1 month after discharge and then every 3-6 months. Clinical outcome was evaluated at discharge and follow-up, according to the modified Rankin Scale score. The
mRS score at the latest clinical follow-up was defined as the final outcome. If the latest clinical follow-up was performed $>3$ months before the commencement of this study, a structured telephone interview was performed to evaluate clinical outcome. ${ }^{15}$ If the patient's clinical condition deteriorated due to an event definitively unrelated to treatment or an intracranial aneurysm, the functional outcome at the follow-up just before that event was defined as the final outcome.

Immediate angiographic results were assessed according to the Raymond classification: complete, neck remnant, or sac remnant. ${ }^{16}$ Follow-up imaging (conventional angiography and/or MR angiography) was performed initially at 6-12 months and then at 24-36 months. Imaging follow-up results were classified into 3 categories compared with immediate posttreatment control angiography: 1 ) stable or improved; 2) minor recurrence, when the recurrence did not require retreatment; and 3) major recurrence, when the recurrence required retreatment. If major recanalization was detected, retreatment was performed.

\section{Statistical Analysis}

All statistical analyses were performed by using the Statistical Package for the Social Sciences, Version 20 (IBM, Armonk, New York). All categoric variables are presented as a percentage and 95\% confidence intervals. All continuous variables are presented as mean $\pm \mathrm{SD}$.

\section{RESULTS}

The characteristics of the patients and aneurysms as well as the clinical and angiographic outcomes are summarized in the Table. One-stage coiling was used to treat 167 patients with $\geq 2$ aneurysms. The patients had 418 aneurysms (2.5 aneurysms per patient); 108 patients had two, 44 had three, 9 had four, 3 had five, 2 had 6, and 1 patient had 7 aneurysms. One-stage coiling was attempted on 359 aneurysms (2.1 aneurysms per patient). In 131 patients (78.4\%), all detected aneurysms were treated with coiling only. Ninety-seven patients were treated with 1 type; 68 patients, with 2 types; and 2 patients, with 3 types of coiling techniques. Specifically, coiling was completed with a single catheter in 151 $(42.1 \%)$, multicatheters in $60(16.7 \%)$, balloon-assisted in 70 (19.5\%), and stent-assisted in 78 (21.7\%) aneurysms. Coiling was aborted in $6(1.7 \%)$ aneurysms due to technical difficulties, but without complications.

The rate of any complication was $6.6 \%(11 / 167)$. Of the 11 complications, 2 occurred in patients with $\mathrm{SAH}$, while the other 9 occurred in patients without SAH. All complications occurred during the treatment of the last aneurysm or were detected after completion of the embolization. Specifically, thromboembolic 

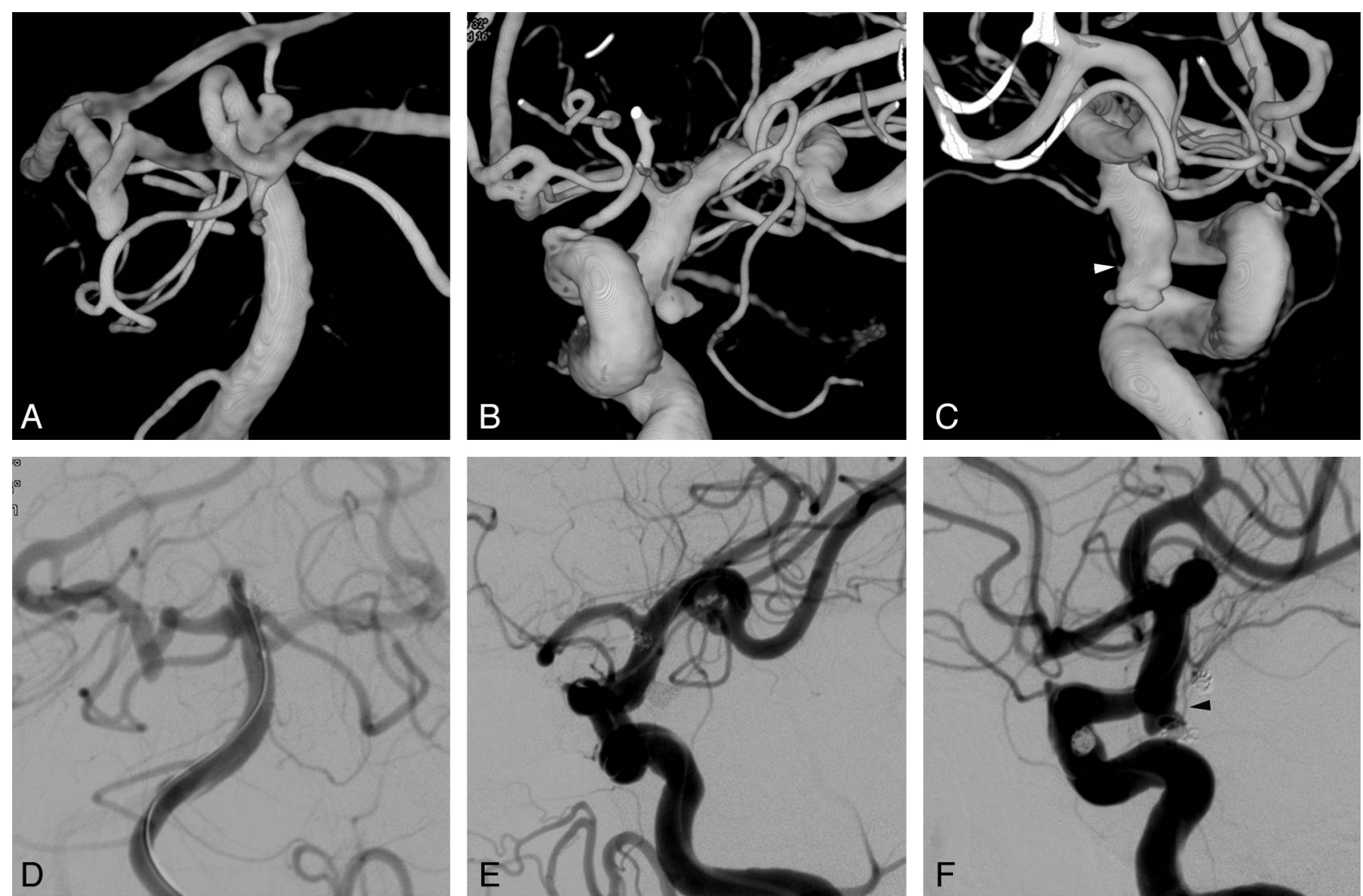

FIG 1. A 61-year-old woman presented with a Hunt and Hess scale grade 3 subarachnoid hemorrhage. Nonenhanced CT (not shown) showed a diffuse, nonlocalized, subarachnoid hemorrhage in the entire basal cistern. 3D reconstruction images of cerebral angiography reveal 3 similarsized small aneurysms at the left superior cerebellar artery origin $(A)$ and bilateral posterior communicating artery origin $(B$ and $C$ ). Each of the 3 aneurysms also has an irregular shape with a daughter sac. The white arrowhead indicates a left tuberothalamic artery incorporated into the aneurysm sac. Postembolization control angiograms after balloon-assisted coiling for all 3 aneurysms show complete occlusion of the superior cerebellar artery $(D)$ and the left posterior communicating artery $(E)$ aneurysms, and an intentional neck remnant, from which a left tuberothalamic artery (black arrowhead) directly originated, of the right posterior communicating artery aneurysm (F).

events occurred in 6 patients, all of which resolved with intraarterial infusion of a glycoprotein IIb/IIIa inhibitor (tirofiban, $0.5-1.0 \mathrm{mg})$. Five of the 6 patients had no symptoms posttreatment, but 1 patient experienced morbidity at discharge (mRS 3) due to a small infarction in the midbrain. After completion of the treatment without any problems during the procedure, perforator infarctions ( 2 brain stem and 1 basal ganglia) were detected in 3 patients, resulting in morbidity at discharge in 2 patients (mRS 2 and 3, respectively). One patient had a puncture site pseudoaneurysm, requiring surgical repair. The final patient had an ICA rupture during balloon-assisted coiling for the last hypophyseal aneurysm after successful embolization of basilar artery tip and anterior choroidal aneurysms, resulting in mortality.

Treatment-related morbidity and mortality at discharge occurred in $3(1.8 \%)$ and in $1(0.6 \%)$ of the 167 patients, respectively. Favorable outcomes (mRS 0-2) at discharge occurred in $129(97.7 \%)$ of 132 patients without SAH and in $27(77.1 \%)$ of 35 patients with SAH. Of the 162 patients (97\%) for whom clinical follow-up was available for a mean of 35.8 months, 154 patients (95.1\%) had favorable outcomes. Immediate posttreatment angiography showed complete occlusion in 186 (51.8\%) patients, neck remnant in 134 (37.3\%), sac remnant in 29 (7.8\%), and failure in $6(1.7 \%)$. Of the $262(73.9 \%)$ aneurysms that had available follow-up imaging for a mean of 24.8 months, $244(93.1 \%)$ showed a stable or improved state and there were 12 (4.6\%) minor and $6(2.3 \%)$ major recurrences. All major recurrent aneurysms were retreated with coiling without complications.

\section{DISCUSSION}

Multiple intracranial aneurysms occur in 19\%-34\% of patients who present with SAH. ${ }^{2-5}$ Coiling as a treatment for multiple intracranial aneurysms has not been well studied. To our knowledge, only 2 small case series of 1-stage coiling for multiple intracranial aneurysms have been reported in the literature. Solander et $\mathrm{al}^{13}$ treated 38 patients with 93 aneurysms with coiling $(n=79)$ and clipping $(n=14)$. Twenty-five patients underwent 1-stage coiling for $>1$ aneurysm at various distributions. No patient had treatment-related permanent morbidity. Xavier et $\mathrm{al}^{14}$ treated 6 patients with 1-stage coiling for 13 aneurysms. No periprocedural complications were reported in that study. In the current study, 1-stage coiling was completed with 1-3 different types of coiling techniques in 353 (98.3\%) of 359 aneurysms in 167 patients, with $1.8 \%$ morbidity and $0.6 \%$. mortality. Furthermore, in $78.4 \%$ of patients, all detected aneurysms were treated with coiling only.

There have been many reported cases in which multiple aneurysms have been treated with clipping. ${ }^{7-12}$ Clipping of multiple aneurysms, however, results in poorer outcomes than in a single aneurysm. ${ }^{7-11}$ This can be explained by the increased manipula- 
tion of cerebral arteries and brain tissue during multiple-aneurysm surgery. ${ }^{8,11}$ Coiling for multiple aneurysms, however, does not require increased manipulation of cerebral arteries or brain tissue. Although the ruptured aneurysm should be treated first, in a patient with SAH who has multiple intracranial aneurysms, it is often difficult to determine which aneurysm is ruptured. In this case, all of the possibly ruptured aneurysms should be treated in the same session. One-stage clipping for multiple aneurysms may be limited in cases in which multiple aneurysms are distributed bilaterally or are in both the anterior and posterior circulations. ${ }^{8}$ One-stage treatment of multiple aneurysms distributed bilaterally or in both the anterior and posterior circulations may be more feasible with coiling than with clipping.

In patients with $\mathrm{SAH}$ who had multiple aneurysms and in whom it was unclear which aneurysm was ruptured, 1-stage coiling for all of the possibly ruptured aneurysms, regardless of the distribution of multiple aneurysms, was an important advantage (Fig 1). In patients with unruptured aneurysms, multiplicity is associated with a greater risk of rupture compared with single aneurysms. ${ }^{17}$ Which patients with unruptured aneurysms will experience $\mathrm{SAH}$ is often unpredictable, and it is even more difficult to determine which of the multiple unruptured aneurysms will rupture. In our institution, 3 patients had SAH from a remaining aneurysm after the most dangerous-looking aneurysms had been treated (data not shown). Therefore, it is our policy to treat, if feasible, all unruptured aneurysms in patients in good medical condition. Because coiling of unruptured aneurysms is associated with less morbidity and mortality than clipping, ${ }^{18}$ it seems reasonable to treat as many aneurysms as possible with coiling.

Another advantage of 1-stage coiling of multiple intracranial aneurysms is that coiling avoids repeat general anesthesia and groin punctures compared with multiple separate procedures; 1-stage coiling may decrease the rate of complications associated with repeat general anesthesia and multiple femoral artery punctures. One-stage coiling of multiple aneurysms may prolong the time that patients remain under general anesthesia. Therefore, in terms of general anesthesia, one should balance the benefits and the risks of 1-stage coiling versus coiling in multiple separate sessions, considering each patient's medical condition.

Cost-effectiveness is also an expected advantage of coiling. Coiling was associated with significantly shorter lengths of hospital stay and significantly lower total hospital charges for patients with both ruptured and unruptured aneurysms, compared with clipping. ${ }^{19}$ One-stage coiling of multiple aneurysms may further shorten the lengths of overall hospital stays and reduce overall total medical costs.

In addition to the retrospective nature, this study has several limitations. Because the treatment strategy was different between the 2 hospitals, case selection for 1-stage coiling was also different, which might affect our results. Coiling technique and perioperative medications have changed during the 10 years of the caseregistration period; these changes might affect angiographic and clinical outcomes. Nonetheless, the results of this study may help determine treatment strategies for multiple intracranial aneurysms.

\section{CONCLUSIONS}

One-stage coiling of multiple intracranial aneurysms seems to be a safe and effective treatment with low morbidity and mortality.

Disclosures: Dong Joon Kim—UNRELATED: Grants/Grants Pending: Korea Healthcare Technology R\&D Project, Yonsei University College of Medicine Faculty research grant.

\section{REFERENCES}

1. Nieuwkamp DJ, Setz LE, Algra A, et al. Changes in case fatality of aneurysmal subarachnoid hemorrhage over time, according to age, sex, and region: a meta-analysis. Lancet Neurol 2009;8:635-42

2. Kassell NF, Torner JC, Jane JA, et al. The international cooperative study on the timing of aneurysm surgery. Part 2. Surgical results. J Neurosurg 1990;73:37-47

3. Kaminogo M, Yonekura M, Shibata S. Incidence and outcome of multiple intracranial aneurysms in a defined population. Stroke 2003;34:16-21

4. Rinne J, Hernesniemi J, Puranen M, et al. Multiple intracranial aneurysms in a defined population: prospective angiographic and clinical study. Neurosurgery 1994;35:803-08

5. Juvela S. Risk factors for multiple intracranial aneurysms. Stroke 2000;31:392-97

6. Andaluz N, Zuccarello M. Recent trends in the treatment of cerebral aneurysms: analysis of a nationwide inpatient database. J Neurosurg 2008;108:1163-69

7. Mount LA, Brisma R. Treatment of multiple intracranial aneurysms. J Neurosurg 1971;35:728-30

8. Mizoi K, Suzuki J, Yoshimoto T. Surgical treatment of multiple aneurysms: review of experience with 372 cases. Acta Neurochir (Wien) 1989;96:8-14

9. Inagawa T. Surgical treatment of multiple intracranial aneurysms. Acta Neurochir (Wien) 1991;108:22-29

10. Vajda J. Multiple intracranial aneurysms: a high risk condition. Acta Neurochir (Wien) 1992;118:59-75

11. Rinne J, Hernesniemi J, Niskanen M, et al. Management outcome for multiple intracranial aneurysms. Neurosurgery 1995;36: 31-37, discussion 37-38

12. Hong $T$, Wang Y. Unilateral approach to clip bilateral multiple intracranial aneurysms. Surg Neurol 2009;72 (suppl 1):S23-28, discussion S28

13. Solander S, Ulhoa A, Vinuela F, et al. Endovascular treatment of multiple intracranial aneurysms by using Guglielmi detachable coils. J Neurosurg 1999;90:857-64

14. Xavier AR, Rayes M, Pandey $P$, et al. The safety and efficacy of coiling multiple aneurysms in the same session. J Neurointerv Surg 2012;4:27-30

15. Janssen PM, Visser NA, Dorhout Mees SM, et al. Comparison of telephone and face-to-face assessment of the modified Rankin Scale. Cerebrovasc Dis 2010;29:137-39

16. Roy D, Milot G, Raymond J. Endovascular treatment of unruptured aneurysms. Stroke 2001;32:1998-2004

17. Sonobe M, Yamazaki T, Yonekura M, et al. Small unruptured intracranial aneurysm verification study: SUAVs study, Japan. Stroke 2010;41:1969-77

18. Higashida RT, Lahue BJ, Torbey MT, et al. Treatment of unruptured intracranial aneurysms: a nationwide assessment of effectiveness. AJNR Am J Neuroradiol 2007;28:146-51

19. Hoh BL, Chi YY, Lawson MF, et al. Length of stay and total hospital charges of clipping versus coiling for ruptured and unruptured adult cerebral aneurysms in the Nationwide Inpatient Sample database 2002 to 2006. Stroke 2010;41:337-42 